\title{
Bullous systemic lupus erythematosus associated with increased activity of lupus nephritis: a case report and review of the literature
}

\author{
Magdalena Żychowska, Aleksandra Batycka-Baran, Joanna Maj, Wojciech Baran \\ Department of Dermatology, Venereology and Allergology, Wroclaw Medical University, Wroclaw, Poland \\ Adv Dermatol Allergol 2018; XXXV (4): 431-433 \\ DOI: https://doi.org/10.5114/ada.2018.77676
}

Bullous systemic lupus erythematosus (BSLE) is a rare subepidermal blistering disease with distinctive histological and immunological patterns. The disease affects mainly young females. The typical presentation is vesicobullous eruption with an acute onset and predilection for the face, trunk, upper extremities and vermillion border [1].

Bullous systemic lupus erythematosus can be the initial presentation of SLE or it may be associated with SLE flare $[2,3]$. The association between BSLE and increased activity of lupus nephritis has been particularly underscored by several authors [4-9]. Herein, we present a patient diagnosed with lupus nephritis several years earlier, in whom the development of BSLE was associated with the recurrence of renal disease.

A 22-year-old woman was admitted to the Department of Dermatology in January 2016 for evaluation of disseminated asymptomatic erythematous lesions with numerous small vesicles, which had developed one month prior to hospitalization. The patient had been diagnosed with lupus nephritis in 2006 and since then treated with systemic corticosteroids, eventually tapered off to a maintenance dose of $4 \mathrm{mg} /$ day of methylprednisolone. No skin lesions had been present in the past. The routine blood cell count and urine analysis, performed one month prior to the development of skin lesions, had been within normal limits and there had been no symptoms of SLE activity. On admission, physical examination revealed disseminated erythematous lesions with numerous tense fluid-filled vesicles, erosions and crusts located on the face, neck, upper part of the trunk and upper extremities (Figures 1 A, B). Nikolsky's sign was negative. No malar rash or discoid rash were present. Laboratory investigations revealed a decreased concentration of hemoglobin $(10.6 \mathrm{~g} / \mathrm{dl})$ and red blood cells count (3.61 $\left.\times 10^{6} / \mu \mathrm{l}\right)$, hematuria with proteinuria (3.3 g/day), hypo- proteinemia (4.8 g/dl; normal range: $6.4-8.3 \mathrm{~g} / \mathrm{dl}$ ) with hypoalbuminemia $(2.7 \mathrm{~g} / \mathrm{dl}$, normal range: $3.5-5.2 \mathrm{~g} /$ $\mathrm{dl})$, decreased levels of C3 (0.252 g/l; normal range: $0.9-$ $1.8 \mathrm{~g} / \mathrm{l})$ and C4 (0.0358 g/l; normal range: 0.1-0,4 g/l) and presence of antinuclear antibodies (ANA), including antidouble-stranded DNA (anti-dsDNA) antibodies. Direct immunofluorescence showed markedly positive granular deposits of IgM, Clq and C3 with discrete IgG and IgA at the dermoepidermal junction. No circulating antibodies were detected on indirect immunofluorescence. Subepidermal blister and neutrophilic infiltrate in the upper dermis were present on histopathological examination (Figure 2). The diagnosis of BSLE with renal involvement was established. The dose of methylprednisolone was increased from a maintenance dose of $4 \mathrm{mg} /$ day to $16 \mathrm{mg} /$ day and chloroquine at a dose of $250 \mathrm{mg} /$ day was introduced, which resulted in visible alleviation of bullous eruption. The patient was referred to the Department of Nephrology for treatment of lupus nephritis.

Despite the high prevalence of cutaneous lesions in SLE (59-85\%), BSLE affects less than 5\% of patients with SLE and is rarely described in the literature $[1,6]$. The disease is caused by autoantibodies to the basement membrane zone (BMZ) antigens, mainly type VII collagen, which is a major component of the anchoring fibrils. However, other target antigens, including laminin 5, laminin 6 and bullous pemphigoid antigen BP230, have also been described. Typical histological findings are subepidermal blisters, neutrophil-predominant infiltrate in the papillary dermis and large deposits of mucin in the reticular dermis [10].

Camisa and Sharma [11] proposed that the diagnosis of BSLE should be based on: 1) fulfilment of the SLE diagnostic criteria; 2) presence of blisters on both sun-exposed and non-sun-exposed skin; 3) presence of IgG and/or IgM

Address for correspondence: Magdalena Żychowska MD, Department of Dermatology, Venereology and Allergology, Wroclaw Medical University, 1 Chałubińskiego St, 50-368 Wroclaw, Poland, phone: +48 669 422 237, e-mail: magda.zychowska@gmail.com Received: 1.05.2017, accepted: 7.05.2017. 

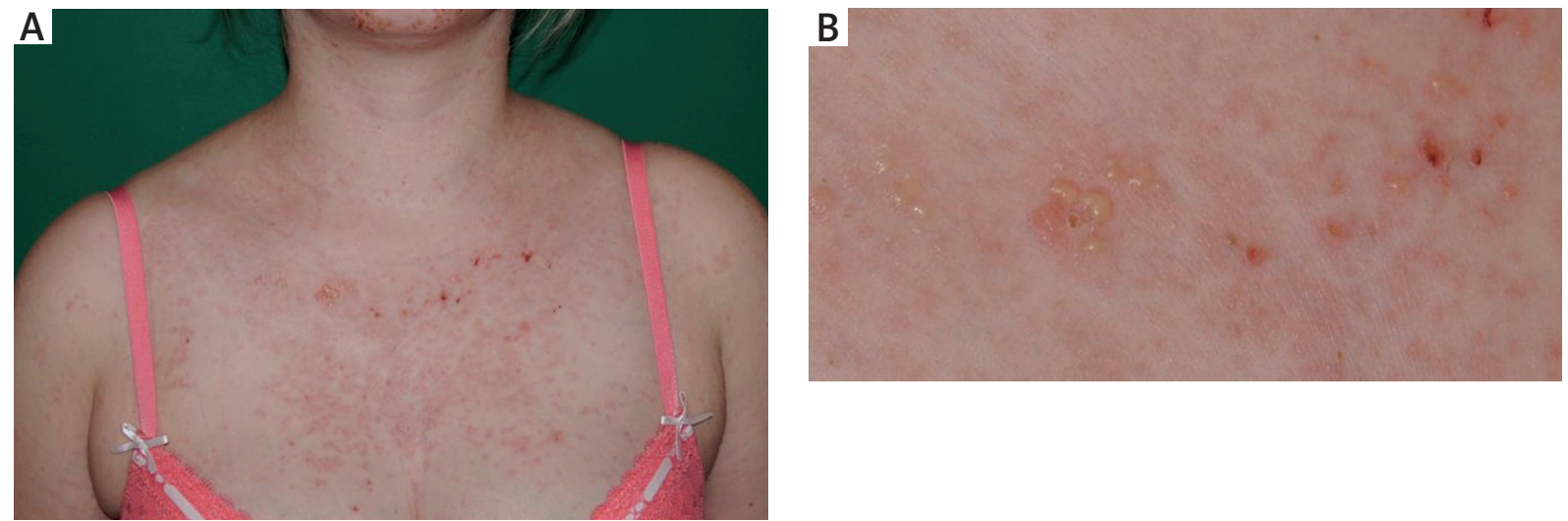

Figure 1. A - Disseminated erythematous plaques with numerous small vesicles, B - tense fluid-filled vesicles over erythematous and normal-appearing skin

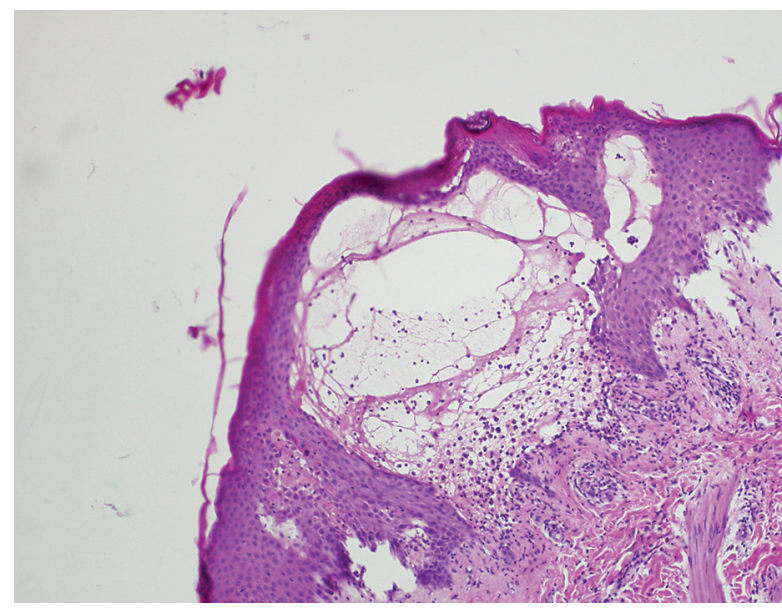

Figure 2. Histopathological image showing a subepidermal blister with inflammatory infiltration in the dermis

and often IgA at the BMZ; 4) histopathological image of the subepidermal blister with neutrophilic infiltrate in the dermis; and 5) presence of antibodies to type VII collagen.

Currently, BSLE is considered a more heterogeneous disease than it was initially believed. Yell et al. [12] revised the diagnostic criteria and distinguished 3 types of BSLE. Type 1 embraces all the cases with detected antibodies to type VII collagen, which bind to the dermal side of the salt-split skin on indirect immunofluorescence. Type 2 consists of patients with negative antibodies to type VII collagen, but fulfilling all the other criteria of BSLE. The antigen in this group of patients has not been precisely identified yet, however, there is a positive reaction with the dermal side of the salt-split skin on indirect immunofluorescence. In type 3, autoantibodies bind to the epidermal side of the salt-split skin [11]. Patients with type 1 BSLE should fulfil all five criteria introduced by Camisa et al., whereas the first four criteria are required for the diagnosis of type 2 or type 3 BSLE.
Camisa and Sharma were the first ones to emphasize the potential association between BSLE and glomerulonephritis [11]. Moreover, they found a high incidence of IgA deposits along the basement membrane zone in patients with BSLE. Since then, an increasing number of BSLE cases associated with lupus nephritis has been published [5-9]. Nevertheless, the exact mechanism underlying this association has not been identified yet.

We would like to contrast BSLE with much more common "SLE with blisters", in which the formation of blisters is not associated with circulating antibodies to type VII collagen, but is a result of severe inflammation with necrosis of keratinocytes or hydropic degeneration of the basal layer. It can affect up to 5\% of patients diagnosed with SLE. Skin lesions in SLE with blisters can mimic toxic epidermal necrolysis or erythema multiforme and bullae always occur over the erythematous skin [5]. The occurrence of blisters on the normal skin should always arouse suspicion of BSLE.

According to data from the literature, dapsone is the treatment of choice. Effectiveness of low doses of dapsone (25-50 mg/day) and striking improvement within 1-2 days have been observed [13]. The response to systemic corticosteroids in monotherapy is usually limited and even paradoxical flare after steroid administration has been reported [14]. Other treatment options include antimalarials, azathioprine, cyclophosphamide, mycophenolate mofetil and, in refractory cases, rituximab [1, 5, 13, 15].

The presented case supports the hypothesis that BSLE may be an indicator of increased activity of SLE, especially lupus nephritis. BSLE poses a diagnostic challenge and it should be considered in any patient with bullous eruption, especially with a history of SLE.

\section{Conflict of interest}

The authors declare no conflict of interest. 


\section{References}

1. Contestable JJ, Edhegard KD, Meyerle JH. Bullous systemic lupus erythematosus: a review and update to diagnosis and treatment. Am J Clin Dermatol 2014; 15: 517-24.

2. Tincopa M, Puttgen KB, Sule S, et al. Bullous lupus: an unusual initial presentation of systemic lupus erythematosus in an adolescent girl. Pediatr Dermatol 2010; 27: 373-6.

3. Mekouar F, Hammi S, Elomri N, et al. Bullous systemic lupus erythematosus. Intern Med 2011; 50: 1445.

4. Camisa C. Vesiculobullous systemic lupus erythematosus. A report of four cases. J Am Acad Dermatol 1988; 18: 93-100.

5. Momen T, Madihi Y. Bullous systemic lupus erythematosus and lupus nephritis in a young girl. Oman Med J 2016; 31: 453-5.

6. Lourenco DM, Gomes RC, Aikawa NE, et al. Childhood-onset bullous systemic lupus erythematosus. Lupus 2014; 23: 1422-5.

7. Jira M, Elgatni M, Sekkach Y, et al. Three cases of bullous lupus erythematosus. Ann Dermatol Venereol 2013; 140: 778-83.

8. Poojary S, Rais S. Bullous systemic lupus erythematosus with lupus nephritis: a rare case of a subepidermal bullous disorder in a child. Cutis 2012; 89: 17-21.

9. Vijayalakshmi AM, Jayavardhana A. Bullous systemic lupus erythematosus and lupus nephritis in a 10 year old boy. Indian Pediatr 2007; 44: 861-3.

10. Vassileva S. Bullous systemic lupus erythematosus. Clin Dermatol 2004; 22: 129-38.

11. Camisa C, Sharma HM. Vesiculobullous systemic lupus erythematosus. Report of two cases and a review of the literature. J Am Acad Dermatol 1983; 9: 924-33.

12. Yell JA, Allen J, Wojnarowska F, et al. Bullous systemic lupus erythematosus: revised criteria for diagnosis. Br I Dermatol 1995; 132: 921-8.

13. Hall RP, Lawley TJ, Smith HR, et al. Bullous aruption of systemic lupus erythematosus. Dramatic response to dapsone therapy. Ann Intern Med 1982; 97: 165-70.

14. Lalova A, Pramatarov K, Vassileva S. Facial bullous systemic lupus erythematosus. Int I Dermatol 1997; 36: 369-71.

15. Alsanafi S, Kovarik C, Mermelstein AL, et al. Rituximab in the treatment of bullous systemic lupus erythematosus. J Clin Rheumatol 2011; 17: 142-4. 